\section{Support for atmospheric origin of anomalous submillimetre wave spectral features}

A RECENT letter in Nature ${ }^{1}$ showed a feature at a frequency of $8 \mathrm{~cm}^{-1}$ in one of two spectra of the atmosphere. That the feature is an artefact follows from the authors' own statement that no true spectral observation was possible. The indication was found only in records from daylight hours and the proposal was made that it was caused by some solar process with an appropriate time variation coupling to the recording system in some unspecified way. We do not wish to comment on this process or on the authors' own evidence given to support it. But they also quote published results as giving further support to their hypothesis with the clear implication that features in the spectra of other workers may be due to the same kind of artcfact. We contest this since it is easy to distinguish between a true spectral indication and an artefact, coming from temporal variations of source or absorption intensity, by varying the time rate of change of path difference given by the interferometer.

TamLe 1 Properties of spectra in Fig. 1

\begin{tabular}{lccc}
\hline & Curve $A$ & Curve $B$ & Curve $C$ \\
Precipitable water $(\mathrm{mm})$ & 0.85 & 0.85 & 1.84 \\
Path length (m) & 80 & 80 & 20.25 \\
Temperature (K) & 293 & 293 & 328 \\
Resolution (cm-1) & 0.25 & 0.25 & 0.5 \\
Transmission value at $8 \mathrm{~cm}^{-1}$ & 0.95 & 0.75 & 0.60 \\
\hline
\end{tabular}

Baluteau et al. cite evidence (sce ref 2 ) with the mistaken supposition that the results came from measurements using the Sun. It was clearly stated that spectra were obtained using an artifical source and a horizontal atmospheric path and, to add to the record, the spectra were recorded in the hours of darkness. The argument for this work sup-

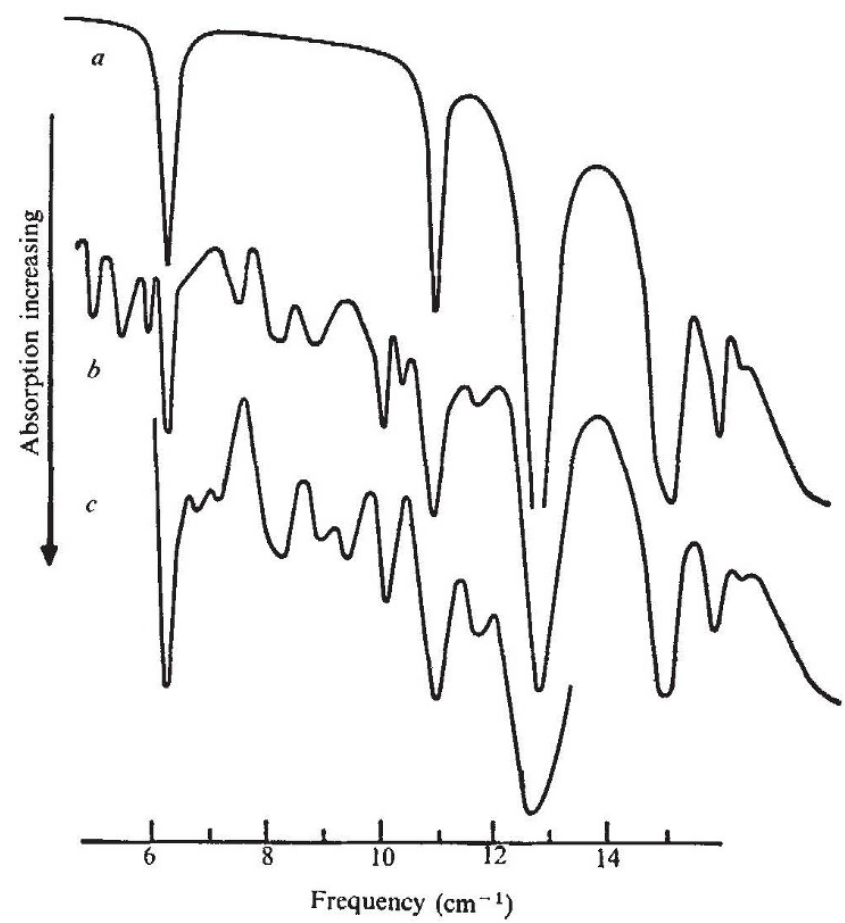

Fig. 1 Water vapour absorption spectra. a, Theoretical spectrum for monomeric water. $b$, Measured spectrum for a horizontal path in the atmosphere. $c$, Laboratory spectrum for water vapour alone. See Table 1. porting their hypothesis is therefore invalid.

In stating that "effectively laboratory spectra" do not show any anomalous peak in this spectral region Baluteau et al. seem to have overlooked other work of ours (ref. 3). Though this gave somewhat lightweight evidence it seems to have been essentially correct and provided an important clue about anomalous absorption. New work which relates to it is shown in Fig. 1 curve $c$.

The topic of anomalous millimetre and submillimetre absorption is being closely studied at the Appleton Laboratory. The two observed spectra (Fig. 1 curves $b$ and $c$; Table 1) which are taken from work which will later be published in full, support our view that anomalous absorption is an atmospheric phenomenon and that the molecular complexity of water vapour is an important, if not the only factor in explaining it.

This work is part of the programme of the Appleton Laboratory.

H. A. Gebbie

R. J. EMERY

R. A. Bohllander

Appleton Laboratory,

Ditton Park,

Slough SL3 9JX, Bucks

Department of Physics and Astrophysics,

G. G. Gimmestad

University of Colorado, Boulder, Colorado 80302

Received November 12, 1973.

${ }^{1}$ Baluteau, J. P., Bussoletti, E., and Epchtein, N., Nature, 244, 562 (1973).

${ }^{2}$ Gebbie, H. A., Bohlander, R. A., and Pardoe, G. W., Nature, 230, 521 (1971).

${ }^{3}$ Harries, J. E., Burroughs, W. J., and Gebbie, H. A., J. quat. Spectrosc. Radiat. Transfer, 9, 809 (1969).

\section{Safety in the preparation of multimetallic catalysts}

THERE is presently an extensive research effort in the field of catalysis coneerned with alloy systems. Many researchers are preparing and characterising unsupported systems in order to study the metal phasc in the absence of the support. A classical approach to doing this is that of simultaneous chemical reduction of the salts of a given alloy system in aqucous solution. Such reducing agents as hydrazine or sodium borohydride are frequently used. During the course of our work we found that the reduction of ruthenium salts with borohydride solutions gave a 'metal powder' which, when dried, violently exploded on touching it with water and sometimes by simple stimulation with a spatula. Under certain conditions a very unstable and poorly characterised 'ruthenium hydride' is apparently formed. A safer alternative technique is to use aqueous solutions of hydrazine for the chemical reduction of ruthenium-containing solutions.

Along these same lines, supported metal catalysts which contain gold should never be prepared by impregnation of a support with solutions which contain both gold salts and $\mathrm{NH}_{4} \mathrm{OH}$, The dried catalysts contain extremely shocksensitive gold-nitrogen compounds which may explode with the lightest touch.

Corporate Research Laboratories,

J. A. Cusumano

Esso Research and Engineering Company,

PO Box 45,

Linden, New Jersey 07036

Received December 19, 1973. 